Pacific Journal of Mathematics

ONE-DIMENSIONAL NASH GROUPS

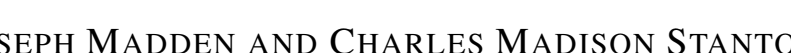




\title{
ONE-DIMENSIONAL NASH GROUPS
}

\author{
James J. Madden and Charles M. Stanton
}

\begin{abstract}
A Lie group equipped with a compatible real algebraic structure is called a locally Nash group. We prove some general facts about locally Nash groups, then we classify the one-dimensional locally Nash groups, using a theorem of Weierstrass that characterizes the analytic functions satisfying an algebraic addition theorem. Besides the standard Nash structure on the additive group of real numbers, there are locally Nash structrures on the additive reals induced by the exponential function, the sine function, and by any elliptic function that is real on $\mathbb{R}$. There are no other simply connected one-dimensional locally Nash groups. Any two quotients of the additive reals with their standard Nash structure by discrete subgroups are Nash equivalent. For other locally Nash structures on $\mathbb{R}$, the quotients $\mathbb{R} / \alpha Z$ and $\mathbb{R} / \beta Z$ are Nash equivalent if and only if $\alpha / \beta$ is rational. The classification of the one-dimensional Nash groups is equivalent to the classification of the one-dimensional semialgebraic groups. It is precisely these groups that are definable over $\mathbb{R}$, so we have also classified the onedimensional groups definable over $\mathbb{R}$.
\end{abstract}

0. Introduction. The main part of this paper concerns semialgebraic geometry and complex analysis, and we expect our audience to be mostly semialgebraic geometers. Nonetheless, our motivation was a problem that arose in mathematical logic. In recent years, logicians have been much interested in questions of the definability of one structure within another. For various reasons, questions of definability within ordered structures have received a lot of attention (see [PS]), as have questions concerning the definability of groups (see [P] and [NP]). These two concerns come together in the papers [R] and [NPR], where groups of low dimension definable in $o$-minimal structures are classified up to group theoretic equivalence. We offer here a classification up to definable equivalence of the one-dimensional groups definable over $\mathbb{R}$.

The results of this paper rest on two important works by others. The first is a theorem of Pillay [P], which reduces the problem of classifying the groups definable over $\mathbb{R}$ to the problem of classifying Nash groups. In other words, despite the fact that not even continuity is assumed in posing the original problem, Pillay's paper shows that we can solve it by working within an analytic category. The second is a 
somewhat neglected theorem of Weierstrass that characterizes the analytic functions on a neighborhood of the origin in the complex plane that satisfy an algebraic addition theorem. The remaining ingredients are some formal lemmas on Nash groups in $\S 2$ and certain considerations that involve $\mathbb{R}$-rationality in $\S 4$. We have organized the paper in such a way that a reader interested only in Nash groups can begin reading at $\S 1$. The applications to definable groups are described in $\S 5$.

The sets definable over $\mathbb{R}$ are exactly the semialgebraic sets. Thus, our theorem is also a classification of the one-dimensional semialgebraic groups up to semialgebraic equivalence. This is merely terminology, but in connection with this there is an important note. In semialgebraic geometry, the functions one considers are almost always continuous. In contrast, in this paper, we do not assume continuity for the group operation and do not assume a semialgebraic group equivalence to be a homeomorphism.

It is worth remarking that although our primary concern is with definable structures, it has proved natural and useful to work with a category including objects that are not definable-namely, the locally Nash groups. These, in general, are definable only locally. Other semialgebraic geometers have noted the convenience of introducing such objects; see, e.g., [DK].

Acknowledgments. We would like to thank A. Pillay for suggesting this problem and for many useful conversations while the paper was being written, M. Shiota for providing a preprint of his book [S] and for useful advice about Nash manifolds, M. Knebusch for valuable comments on an earlier version of the paper, and the referee for a number of useful suggestions.

1. Nash manifolds. A semialgebraic subset of $\mathbb{R}^{n}$ is a finite union of sets of the form

$$
\left\{x \in \mathbb{R}^{n}: f_{1}(x)=\cdots=f_{k}(x)=0, g_{1}(x)>0, \ldots, g_{l}(x)>0\right\}
$$

where $f_{1}, \ldots, f_{k}, g_{1}, \ldots, g_{l}$ are polynomial functions on $\mathbb{R}^{n}$. By Tarski's theorem on the elimination of quantifiers, the semialgebraic sets are precisely the sets definable over $\mathbb{R}$ in the first order language of fields.

A semialgebraic function is a function (necessarily between semialgebraic sets) whose graph is a semialgebraic set.

A Nash function is a real analytic function $f=\left(f_{1}, \ldots, f_{m}\right): U \rightarrow$ $\mathbb{R}^{m}$ (where $U$ is an open semialgebraic subset of $\mathbb{R}^{n}$ ) such that for 
each of the components $f_{k}$ there is a nontrivial polynomial $P$ such that $P\left(x_{1}, \ldots, x_{n}, f_{k}\left(x_{1}, \ldots, x_{n}\right)\right)=0$ for all $\left(x_{1}, \ldots, x_{n}\right) \in U$. Equivalently, a Nash function is a function that is at once analytic and semialgebraic.

Let $M$ be a manifold of dimension $n$. A Nash chart is a homeomorphism $\psi: U \rightarrow S$, where $U$ is an open subset of $M$ and $S$ is an open semialgebraic subset of $\mathbb{R}^{n}$. Two Nash charts $\psi_{i}$ and $\psi_{j}$ with domains $U_{i}$ and $U_{j}$ are Nash compatible if $\psi_{i}\left(U_{i} \cap U_{j}\right)$ is semialgebraic and

$$
\psi_{j} \circ \psi_{i}^{-1}: \psi_{i}\left(U_{i} \cap U_{j}\right) \rightarrow \psi_{j}\left(U_{i} \cap U_{j}\right)
$$

is a Nash diffeomorphism. A locally Nash atlas on $M$ is a set of Nash compatible charts whose domains cover $M$. Equipped with a locally Nash atlas $\left\{\psi_{i}\right\}, M$ is called a locally Nash manifold. A Nash manifold is a locally Nash manifold whose atlas has finitely many charts. A Nash manifold is a definable object. Its underlying set may be taken to be a semialgebraic set, namely the disjoint union of the images of the charts, modulo the definable equivalence relation determined by the gluing maps.

The simplest Nash manifold of dimension $n$ is the manifold $\mathbb{R}^{n}$ with the Nash atlas whose only chart is the identity maping of $\mathbb{R}^{n}$. We shall say that this manifold is $\mathbb{R}^{n}$ with the standard Nash structure.

If $\left(M,\left\{\psi_{i}\right\}\right)$ and $\left(N,\left\{\phi_{j}\right\}\right)$ are locally Nash manifolds, then a Nash mapping is a continuous map $F: M \rightarrow N$ such that for all $i$ and $j$

$$
\phi_{j} \circ F \circ \psi_{i}^{-1}: \psi_{i}\left(F^{-1}\left(V_{j}\right) \cap U_{i}\right) \rightarrow \phi_{j}\left(V_{j}\right)
$$

is a Nash function. (Here, of course, $U_{i}$ and $V_{j}$ are the domains of $\psi_{i}$ and $\left.\phi_{j}.\right)$ A bijective Nash mapping $F: M \rightarrow N$ is a Nash equivalence if the mapping $F^{-1}: N \rightarrow M$ is also Nash. If $\left\{\psi_{i}\right\}$ and $\left\{\phi_{j}\right\}$ are compatible locally Nash atlases for $M$ (i.e., for all $i$ and $j$, $\psi_{i}$ and $\phi_{j}$ are compatible), then clearly the identity map is a Nash equivalence of $\left(M,\left\{\psi_{i}\right\}\right)$ with $\left(M,\left\{\phi_{j}\right\}\right)$.

LEMMA. The composite of two Nash maps is Nash.

Proof. In view of the definitions, this reduces to the known fact that if $f: U \rightarrow \mathbb{R}$ is an analytic function defined on an open semialgebraic subset $U$ of $\mathbb{R}^{n}$ and if, for each $u \in U$, there is a neighborhood $V$ of $u$ on which $f$ is semialgebraic, then $f$ is semialgebraic on $U$. 
REMARK. The lemma is more delicate than one might assume. For example, if we were to define locally $C^{0}$-semialgebraic manifolds in analogy to the way we defined locally Nash manifolds, then the corresponding lemma would fail. The lemma, and the comment immediately preceding it, imply that a map which is Nash with respect to one atlas (on either the domain or codomain) is Nash with respect to any compatible atlas. This is not true in the $C^{0}$ case. A periodic piecewise linear function on $\mathbb{R}$ is $C^{0}$-semialgebraic with respect to the domain atlas whose charts are the identity function on compact intervals and the codomain atlas with a single chart. It is not $C^{0}$-semialgebraic with respect to the compatible domain atlas with but one chart. The lemma and the sentence preceding it show that there is no need to introduce the term "locally Nash map". A map between manifolds which is Nash with respect to given locally Nash atlases on its domain and codomain is Nash with respect to any Nash atlases-finite or not-which are compatible with the given atlases. Another way of making this point would be to say that the category of Nash manifolds is a full subcategory of the category of locally Nash manifolds. Note that the category of semialgebraic spaces and semialgebraic maps is not a full subcategory of the category of spaces locally modeled in the classical sense on locally semialgebraic spaces and maps. (This is slightly different from the category considered in [DK].)

2. Nash groups. If $G$ is a (locally) Nash manifold, then $G \times G$ is a (locally) Nash manifold in a natural way. If $G$ is equipped with group operations

$$
G \times G \rightarrow G ;(x, y) \mapsto x \cdot y \quad \text { and } \quad G \rightarrow G ; x \mapsto x^{-1}
$$

that are given by Nash mappings, then $G$ is called a (locally) Nash group. There is an obvious forgetful functor from (locally) Nash groups to real analytic groups.

We want to consider what data are required in order to determine a locally Nash group atlas on a given real analytic group $G$. Any coordinate chart must be an analytic map. Also, if a locally Nash group atlas on $G$ is given, then an equivalent atlas can be manufactured from any one chart and the group operation, since the translates of any single chart form a locally Nash group atlas.

If $G$ is a real analytic group, then a chart on a neighborhood of the identity that satisfies algebraic compatibility conditions with the group operation in $G$ actually determines a locally Nash group structure on $G$. The following lemma makes this precise. Let $M$ be the graph of 
the group operation $(M=\{(g, h, g h): g, h \in G\})$, and for each $g \in$ $G$, let $K_{g}$ be the graph of conjugation by $g\left(K_{g}=\left\{\left(h, g^{-1} h g\right): h \in\right.\right.$ $G\})$. Then we have

Lemma 1. Suppose $U \subseteq G$ is an open neighborhood of the identity and $\psi$ is an analytic homeomorphism of $U$ with an open semialgebraic subset of $\mathbb{R}^{n}$. If $\psi \times \psi \times \psi(M \cap(U \times U \times U))$ is semialgebraic and for all $g \in G$ there is a neighborhood of the identity $U_{g} \subseteq U$ such that $\psi \times \psi\left(K_{g} \cap\left(U_{g} \times U_{g}\right)\right)$ is semialgebraic, then $\psi$ and its translates determine a locally Nash group atlas on $G$.

Proof. Let $\mathscr{V}$ be the set of all neighborhoods $V$ of the identity such that $V=V^{-1}, V^{2} \subseteq U$, and $\psi(V)$ is semialgebraic. The sets $g V$, where $g \in G$, and $V \in \mathscr{V}$, cover $G$. We show that the maps

$$
\psi_{g}: g V \rightarrow \mathbb{R}^{n} ; \quad g v \mapsto \psi(v)
$$

define a locally Nash group atlas.

If $g V_{1} \cap h V_{2}=W \neq \varnothing$, then the graph of the transition function $\psi_{g}(W) \rightarrow \psi_{h}(W)$ is the projection of the following subset of $\mathbb{R}^{3 n}$ onto the last $2 n$ coordinates:

$$
\begin{gathered}
\psi \times \psi \times \psi(M \cap(U \times U \times U)) \cap\left\{\psi\left(h^{-1} g\right)\right\} \\
\times \psi\left(V_{1}\right) \times \mathbb{R}^{n} \cap\left\{\psi\left(h^{-1} g\right)\right\} \times \mathbb{R}^{n} \times \psi\left(V_{2}\right) .
\end{gathered}
$$

Therefore the function is semialgebraic. As it is analytic, it is Nash, and so we have a locally Nash atlas.

To show that inversion (= inv) is Nash, it suffices to show that

$$
\psi_{g^{-1}} \circ(\text { inv }) \circ\left(\psi_{g}\right)^{-1}
$$

is Nash on $\psi(V)$ for sufficiently small $V \in \mathscr{V}$. This function is equal to the following composition:

$$
\psi_{g}(g v)=\psi(v) \mapsto \psi\left(g v g^{-1}\right) \mapsto \psi\left(g v^{-1} g^{-1}\right)=\psi_{g^{-1}}\left(v^{-1} g^{-1}\right) .
$$

By assumption this composition is Nash when $V \in \mathscr{V}$ is sufficiently small and $v \in V$.

To show that multiplication is locally Nash, it suffices to show that each $\left(g_{1}, g_{2}\right) \in G \times G$ has a neighborhood on which multiplication is Nash. Pick $V_{2} \in \mathscr{V}$ so small that $V_{1}=g_{2} V_{2} g_{2}^{-1} \subseteq U$. Then the following functions are defined and are Nash whenever $z_{i} \in V_{i}$, $i=1,2$, and $h \in g_{1} V_{1} g_{2} V_{2}$ :

$$
\begin{gathered}
\left(\left(\psi_{g_{1}}\left(g_{1} z_{1}\right), \psi_{g_{2}}\left(g_{2} z_{2}\right)\right)=\left(\psi\left(z_{1}\right), \psi\left(z_{2}\right)\right)\right) \mapsto\left(\psi\left(g_{2}^{-1} z_{1} g_{2}\right), \psi\left(z_{2}\right)\right) \\
\mapsto \psi\left(g_{2}^{-1} z_{1} g_{2} z_{2}\right) \mapsto\left(\psi\left(h^{-1} g_{1} g_{2} g_{2}^{-1} z_{1} g_{2} z_{2}\right)=\psi_{h}\left(g_{1} z_{1} g_{2} z_{2}\right)\right) .
\end{gathered}
$$


LEMMA 2. If $G$ is abelian or $G$ is connected, then the hypotheses about the conjugation may be dropped from Lemma 1 .

Proof. This is obvious if $G$ is abelian. If $G$ is connected, then any neighborhood of the origin generates $G$. The existence of $U_{g}$ for all $g \in G$ follows from the fact that conjugation by any element of $U$ is Nash on a sufficiently small neighborhood of the identity.

If $G, U$, and $\psi$ are as in the lemma, then $G$ endowed with the locally Nash structure described above is denoted $(G, \psi)$. If we wish to emphasize the group operation, we write instead $(G, \cdot, \psi)$. For example, the additive group of real numbers with the standard Nash structure is denoted by $(\mathbb{R},+, \mathrm{id})$, where $\mathrm{id}: \mathbb{R} \rightarrow \mathbb{R}$ is the identity function. Suppose an analytic homeomorphism $\phi: U \rightarrow \mathbb{R}^{n}$ satisfies a semialgebraic relation with $\psi$ (i.e., $(\psi, \phi)(U) \subseteq \mathbb{R}^{2 n}$ is semialgebraic). From the properties of semialgebraic sets, it follows that $\phi$ also satisfies the hypotheses of the lemma and that the identity function on $G$ induces an equivalence of locally Nash groups: $(G, \psi) \cong(G, \phi)$. A very simple case of this is used below, namely, if $(\mathbb{R}, \psi)$ is a locally Nash group and $\phi$ is algebraic over $\mathbb{R}[\psi]$, then $(\mathbb{R}, \phi)$ is an equivalent locally Nash group.

3. Weierstrass's theorem. We shall say that a complex function $\phi$ satisfies an algebraic addition theorem on a domain $D \subset \mathbb{C}$ if there is a non-zero polynomial $G(X, Y, Z)$ with complex coefficients such that $G(\phi(x), \phi(y), \phi(x+y))=0$ whenever $x, y$, and $x+y$ belong to $D$. We shall denote by $\mathbb{C}(f)$ the field of all rational functions in $f$. Then we have

THeOREM (Weierstrass). Let $\phi(u)$ be holomorphic in a connected open set containing the origin and satisfy an algebraic addition theorem there. Let $\boldsymbol{\Phi}$ denote the complete analytic function determined by $\phi(u)$. Then one of the following holds.

(1) $\boldsymbol{\Phi}$ is algebraic over $\mathbb{C}(z)$;

(2) $\boldsymbol{\Phi}$ is algebraic over $\mathbb{C}(\exp (\alpha z))$ for some $\alpha$ in $\mathbb{C}$;

(3) $\boldsymbol{\Phi}$ is algebraic over $\mathbb{C}(\wp(z))$ for some Weierstrass $\wp$-function.

Although references to the theorem occur frequently in modern texts on elliptic functions $([\mathbf{C}]$, [Si]) we have been unable to locate a source containing a proof that is easily accessible to the modern reader. Therefore, we have provided a sketch of the proof in an appendix to this paper. 
Suppose that $\phi$ is holomorphic in a disk centered at the origin in $\mathbb{C}$ and that its Taylor series has real coefficients.

We collect for later reference some information about the complete analytic function $\boldsymbol{\Phi}$ determined by $\phi$. Since $\phi(z)$ is real for real $z$, it follows that

$$
\xi(z)=\sum_{n=0}^{\infty} a_{n}(z-a)^{n}
$$

is a function element of $\Phi$ centered at $z=a$ if and only if

$$
\eta(z)=\sum_{n=0}^{\infty} \bar{a}_{n}(z-\bar{a})^{n}
$$

is a function element of $\Phi$ centered at $z=\bar{a}$. We express this fact by saying that the function elements of $\boldsymbol{\Phi}$ at $z=\bar{a}$ are obtained from those at $z=a$ by conjugation. We shall also abuse our language by saying that the function elements

$$
\xi(z)=\sum_{n=0}^{\infty} a_{n}(z-a)^{n} \quad \text { and } \quad \eta(z)=\sum_{n=0}^{\infty} b_{n}(z-b)^{n}
$$

centered at $z=a$ and $z=b$ are the same if $a_{n}=b_{n}$ for all $n$. Suppose that there is a complex number $\omega$ such that for all $a$ in a certain domain $\Phi$ has the same sets of function elements at $z=a$ and $z=a+\omega$. One can then verify that $\Phi$ has the same sets of function elements at $z=\bar{a}$ and $z=\bar{a}+\bar{\omega}$.

4. Results. Let $G$ be a connected locally Nash group. The universal covering group $\pi: \widehat{G} \rightarrow G$ (think of $G$ as a Lie group) becomes a locally Nash group if we obtain Nash coordinate neighborhoods on $\widehat{G}$ by lifting evenly covered Nash coordinate neighborhoods from $G$. If two locally Nash groups are Nash isomorphic, then this isomorphism lifts to a Nash isomorphism of their simply connected covering groups. Thus the classification problem for Nash groups breaks into two parts: (1) determine the isomorphism classes of simply connected locally Nash groups, and (2) for each simply connected Nash group determine the isomorphism classes of quotients of that group by discrete subgroups.

We first consider the simply connected case. Up to isomorphism, the only simply connected one-dimensional Lie group is $(\mathbb{R},+)$, the real numbers with addition and the standard differentiable structure. Thus our task is to classify the Nash structures on $(\mathbb{R},+)$ that make + into a Nash mapping. Using the notation introduced at the end of $\S 2$, we have 
THEOREM 1. Every connected simply connected one-dimensional locally Nash group is equivalent to one of the following locally Nash groups: $(\mathbb{R},+, \mathrm{id}),(\mathbb{R},+, \exp ),(\mathbb{R},+, \sin )$, or $(\mathbb{R},+, \wp)$, where $\wp$ is the Weierstrass $\wp$-function arising from a lattice in $\mathbb{C}$ that is symmetric about $\mathbb{R}$.

REMARK. ( $\mathbb{R},+$, id) is $(\mathbb{R},+)$ with the standard Nash structure, $(\mathbb{R},+, \exp )$ is Nash equivalent to the multiplicative group of positive reals with its standard Nash structure, and $(\mathbb{R},+, \sin )$ is the simply connected connected cover of the unit circle in $\mathbb{C}$, where the circle inherits its Nash structure from the standard Nash structure on $\mathbb{C}$.

Proof of Theorem 1 . Let a locally Nash structure be given on $(\mathbb{R},+)$ and let $\phi: U \rightarrow \mathbb{R}$ be a Nash chart containing 0 . We can choose a subset $V$ of $U$ such that $\phi(V)$ is semialgebraic and $V+V \subset U$. Since the group operation + is a locally Nash mapping, the graph of the mapping $(\phi(x), \phi(y)) \mapsto \phi(x+y)$, where $x, y \in V$, is a semialgebraic set contained in $\phi(V) \times \phi(V) \times \phi(U)$. Hence there is a nontrivial real polynomial $G(X, Y, Z)$ such that

$$
G(\phi(x), \phi(y), \phi(x+y))=0, \quad x, y \in V .
$$

The Taylor series defining $\phi$ gives an extension of $\phi$ to a holomorphic function in a disk $D$ centered at the origin in $\mathbb{C}$. The equation (1) continues to hold in $D$. That is, $\phi$ satisfies an algebraic addition theorem, and so we may appeal to the Weierstrass theorem.

If $\boldsymbol{\Phi}$ is algebraic over $\mathbb{C}(z)$ then the locally Nash structure $\phi$ induces is equivalent to $(\mathbb{R},+$,id). In the other cases, the fact that $\phi$ is real restricts the exponentials or $\wp$-functions with which $\Phi$ may have an algebraic relation.

Suppose $\Phi$ is algebraic over $\mathbb{C}(\exp (\alpha z))$. By the remarks in $\S 3$ the periods of $\exp (\alpha z)$ must be either real or purely imaginary, so $\alpha$ must be either real or purely imaginary. If $\alpha$ is real, the locally Nash structure induced by $\phi$ is equivalent to $(\mathbb{R},+$, exp). (Note that if $\alpha$ is a nonzero real number, the mapping $x \mapsto \alpha x$ is a Nash equivalence between $(\mathbb{R},+, \exp (\alpha z))$ and $(\mathbb{R},+, \exp (z))$. If $\alpha$ is purely imaginary, the locally Nash structure induced by $\phi$ is equivalent to $(\mathbb{R},+, \sin )$.

Suppose $\Phi$ is algebraic over $\mathbb{C}(\wp)$ for some Weierstrass $\wp$-function $\wp$. Then $\Phi$ is a root of the equation

$$
F(X)=0
$$


where $F(X)$ is a polynomial whose coefficients are rational functions of $\wp$ and $\wp^{\prime}$. Let $\Sigma$ be the set of all points in $\mathbb{C}$ at which some coefficient of $F(X)$ has a pole or at which the equation (2) has a multiple root. Let $\Lambda$ be the lattice of periods of the coefficients of $F$. This lattice contains as a sublattice the lattice associated with $\wp$. We shall show that $\Lambda$ is symmetric with respect to the real axis. If $a \notin \Sigma$ and $\omega \in \Lambda$ then the coefficients of $F(X)$ have the same values at $a$ and $a+\omega$. Thus $\Phi$ has the same sets of function elements at $a$ and $a+\omega$. By our remarks in $\S 3, \boldsymbol{\Phi}$ has the same sets of function elements at $\bar{a}$ and $\bar{a}+\bar{\omega}$. The coefficients of $F$ at any point outside $\Sigma$ are the elementary symmetric functions of the values of the function elements of $\boldsymbol{\Phi}$ there. Thus the coefficients at $\bar{a}$ and $\bar{a}+\bar{\omega}$ are the same. Hence $\bar{\omega}$ belongs to $\Lambda$ and so $\Lambda$ is symmetric with respect to the real axis, and $\boldsymbol{\Phi}$ is algebraic over the corresponding field of elliptic functions. We have proved Theorem 1.

We next want to determine the Nash isomorphism classes of examples of simply connected locally Nash groups of elliptic type. Observe first that each isomorphism class contains a locally Nash group $(\mathbb{R},+, \wp)$ with $\wp$ having least real period 1 because the map

$$
(\mathbb{R},+, \wp(\alpha x)) \rightarrow(\mathbb{R},+, \wp(x)) ; \quad x \mapsto \alpha x
$$

is a Nash group isomorphism.

If the least real period of $\wp$ is 1 and $\Lambda$ is symmetric about $\mathbb{R}$ then the lattice for $\wp$ is either of the form $\Lambda_{0}=\mathbb{Z}+i \alpha \mathbb{Z}, \alpha \in$ $\mathbb{R}\left(\Lambda_{0}\right.$ "rectangular"), or of the form $\Lambda_{1}=\mathbb{Z}+\left(\frac{1}{2}+i \alpha\right) \mathbb{Z}, \alpha \in$ $\mathbb{R}$ ( $\Lambda_{1}$ "diamond-shaped"). In the latter case $2 \Lambda_{1}$ is contained in the rectangular lattice $\mathbb{Z}+i \alpha \mathbb{Z}$, and so the Weierstrass function $\wp_{1}$ corresponding to $\Lambda_{1}$ satisfies an algebraic relation with a Weierstrass function having a rectangular lattice.

THEOREM 2. Each Nash equivalence class of locally Nash groups of type $(\mathbb{R},+, \wp)$ is represented by a locally Nash group whose charts are determined by a $\wp$-function whose lattice is of the form $\Lambda=\mathbb{Z}+$ $i \alpha \mathbb{Z}, \alpha \in \mathbb{R}$. Two such groups are Nash equivalent if and only if the ratio of their imaginary periods is rational.

Proof. Only the second statement remains to be shown. Let $\wp_{1}$ and $\wp_{2}$ be associated with the lattices $\mathbb{Z}+i \alpha_{1} \mathbb{Z}$ and $\mathbb{Z}+i \alpha_{2} \mathbb{Z}$, where $\alpha_{1}, \alpha_{2} \in \mathbb{R}$. If $\alpha_{1} / \alpha_{2}$ is rational, then $\wp_{1}$ and $\wp_{2}$ satisfy an algebraic 
relation so the map

$$
\left(\mathbb{R},+, \wp_{1}\right) \rightarrow\left(\mathbb{R},+, \wp_{2}\right) ; \quad x \mapsto x
$$

is a Nash group isomorphism. On the other hand, suppose $\left(\mathbb{R},+, \wp_{1}\right)$ and $\left(\mathbb{R},+, \wp_{2}\right)$ are isomorphic. The isomorphism is given by a dilation $x \mapsto \rho x$ with $\rho \in \mathbb{R}$. Since the real periods of $\wp_{1}$ and $\wp_{2}$ are the same, $\rho \in \mathbb{Q}$. To see this we argue as follows. In terms of the coordinates given by $\wp_{1}$ and $\wp_{2}$ the isomorphism is a Nash mapping, thus there is a real polynomial $F(X, Y)$ such that

$$
F\left(\wp_{1}(x), \wp_{2}(x)\right)=0 .
$$

This equation holds everywhere in $\mathbb{C}$. The equation is unchanged if we replace $x$ by $x+n, n \in \mathbb{Z}$, because $\wp_{1}$ has real period 1 . Thus we see that the set $\left\{\wp_{2}(\rho x+\rho n): n \in \mathbb{Z}\right\}$ is finite, being contained in the set of roots of the polynomial $F\left(\wp_{1}(x), Y\right)$. Since $\wp_{2}$ also has real period 1 the set $\{(\rho x+\rho n) \bmod 1: n \in \mathbb{Z}\}$ is finite and thus so is the set $\{\rho n \bmod 1: n \in \mathbb{Z}\}$. Therefore $\rho$ is rational.

Since $\rho$ is rational there is an algebraic relation between $\wp_{2}(x)$ and $\wp_{2}(\rho x)$ :

$$
F_{1}\left(\wp_{2}(x), \wp_{2}(\rho x)\right)=0
$$

for some polynomial $F_{1}(X, Y)$. We can eliminate $\wp_{2}(\rho x)$ from this relation and (3), obtaining an algebraic relation

$$
F_{2}\left(\wp_{1}(x), \wp_{2}(x)\right)=0
$$

for some polynomial $F_{2}(X, Y)$. An argument similar to the one given above shows that the set $\left\{n \alpha_{1} \bmod \alpha_{2}: n \in \mathbb{Z}\right\}$ is finite, thus the ratio $\alpha_{1} / \alpha_{2}$ must be rational. This completes the proof of Theorem 2 .

In order to complete the classification of one-dimensional Nash groups, we must deal with the quotients of the simply connected groups. Since these are compact, they are Nash groups. These are all of the form $(\mathbb{R},+, \phi) / \alpha \mathbb{Z}$, with $\phi$ as above and $\alpha$ a positive real number. Let $\alpha$ and $\beta$ be any positive reals and consider the map

$$
m:(\mathbb{R},+, \phi) / \alpha \mathbb{Z} \rightarrow(\mathbb{R},+, \phi) / \beta \mathbb{Z}
$$

induced by $x \mapsto \beta x / \alpha$. Clearly $(\mathbb{R},+, \phi) / \alpha \mathbb{Z}$ and $(\mathbb{R},+, \phi) / \beta \mathbb{Z}$ are Nash equivalent groups if and only if $m$ is a Nash map. If $\phi=\mathrm{id}$, then $m$ is Nash regardless of $\alpha$ and $\beta$. In every other case $m$ is Nash if and only if $\alpha / \beta$ is a rational number, since $\phi \circ m \circ \phi^{-1}$ is Nash only in this case. 
Chillingworth and Hubbard $[\mathbf{C H}]$ have shown that the underlying Nash manifolds of $(\mathbb{R},+, \sin ) / \alpha \mathbb{Z}$ and $(\mathbb{R},+, \sin ) / \beta \mathbb{Z}$ are Nash equivalent if and only if $\alpha / \beta$ is a rational number and that when $\alpha$ is not a rational multiple of $\pi,(\mathbb{R},+, \sin ) / \alpha \mathbb{Z}$ is not Nash embeddable in any $\mathbb{R}^{n}$. (See $[\mathbf{C H}]$ or $[\mathbf{S}]$ for a complete discussion of "embeddable" Nash manifolds. In [S], these are called "affine Nash manifolds".) Their argument applies without modification to $(\mathbb{R},+, \wp) / \alpha \mathbb{Z}$. As a Nash manifold, this is embeddable in some $\mathbb{R}^{n}$ if and only if $\alpha$ is a rational multiple of the real period of $\wp$. The image will be a component of the set of real points of a real elliptic curve. Note that the Nash manifolds $(\mathbb{R},+$, id $) / \mathbb{Z}$ and $(\mathbb{R},+$, exp) $/ \beta \mathbb{Z}$ are not embeddable. For if they were-say in $\mathbb{R}^{n}$-the covering maps would induce periodic Nash functions on $\mathbb{R}$. But this is impossible.

We summarize these results in the following:

THEOREM. Every connected one-dimensional Nash group is equivalent, as a Nash group, to one of the following:

(1) $(\mathbb{R},+$, id);

(2) $(\mathbb{R},+, \exp )$;

(3) $(\mathbb{R},+$, id $) / \mathbb{Z}$;

(4) $(\mathbb{R},+, \exp ) / \beta \mathbb{Z}$;

(5) $(\mathbb{R},+, \sin ) / \beta \mathbb{Z}$;

(6) $\left(\mathbb{R},+, \wp_{\alpha}\right) / \beta \mathbb{Z}$.

(Here $\wp_{\alpha}$ denotes the Weierstrass elliptic function with period lattice $\mathbb{Z}+i \alpha \mathbb{Z}, \alpha \in \mathbb{R}$.)

If $\phi \neq \mathrm{id}$, then the Nash groups $(\mathbb{R},+, \phi) / \beta \mathbb{Z}$ and $(\mathbb{R},+, \phi) / \beta^{\prime} \mathbb{Z}$ are isomorphic as Nash groups if and only if $\beta / \beta^{\prime}$ is rational.

The embeddable Nash groups are $(\mathbb{R},+, \mathrm{id}),(\mathbb{R},+$, exp $)$, the groups of type (5) with $\beta$ a rational multiple of $\pi$, and the groups of type (6) with $\alpha / \beta$ rational.

5. Semialgebraic groups and Nash groups. The purpose of this section is to describe how our results on Nash groups apply also to semialgebraic groups. The main point is that there is an equivalence of categories between semialgebraic groups and Nash groups. We briefly sketch the ideas involved.

A semialgebraic group is a semialgebraic set $G \subset \mathbb{R}^{n}$ equipped with a group operation $*: G \times G \rightarrow G$ whose graph is a semialgebraic subset of $\mathbb{R}^{3 n}$. In other words, * is a semialgebraic map. It is not required that $*$ be continuous with respect to the topology that $G$ inherits 
from $\mathbb{R}^{n}$. For example, $G=[0,1)$ equipped with the operation $a * b=a+b-\lfloor a+b\rfloor$ is a semialgebraic group.

Pillay has shown [P, Remark 2.6] that if $G$ is a semialgebraic group, then it is possible to find a finite collection of open semialgebraic subsets $U_{i} \subset \mathbb{G}$ and semialgebraic charts $\psi_{i}: U_{i} \rightarrow \mathbb{R}^{n}$ that induce on $G$ the structure of a Nash group. Following the ideas of that paper, one readily shows that if $\Theta: G \rightarrow G$ is a semialgebraic group homomorphism, then $\Theta$ is a Nash homomorphism with respect to the induced Nash structures on $G$ and $H$. In this way one gets a functor from semialgebraic groups to Nash groups.

On the other hand, if $G$ is a Nash group, then $G$ may be regarded as a semialgebraic group in the following way: If $\left\{\psi_{i}: U_{i} \rightarrow \mathbb{R}^{n}\right\}$ is a Nash atlas for $G$, then the underlying set of $G$ may be identified with $\bigcup \psi_{i}\left(U_{i}\right)$ modulo the equivalence relation induced by the overlaps of the neighborhoods. This is a "semialgebraic space" (see [DK]), and it is regular because $G$ is a topological group. Therefore, by Robson's embedding Theorem [Ro], $G$ may be identified, by a semialgebraic map, with a semialgebraic subset of some $\mathbb{R}^{m}$. This identification, of course, will generally fail to be a Nash mapping.

Appendix: The Weierstrass characterization. In this section we describe Weierstrass's characterization of functions satisfying algebraic addition theorems. We shall follow the development in [H]. (The authors have written a note containing a detailed version of the proof in more modern language. This note is available to any reader who desires it.) It is convenient first to describe meromorphic functions in the complex plane $\mathbb{C}$ that satisfy algebraic addition theorems. We shall denote by $\mathbb{C}(f, g, \ldots)$ the field of rational functions in $f, g, \ldots$. We have

THEOREM (Weierstrass). Let $\phi(u)$ be meromorphic in the complex plane $\mathbb{C}$ and satisfy an algebraic addition theorem there. Then one of the following holds:

(1) $\phi(u)$ belongs to $\mathbb{C}(z)$, i.e., $\phi(u)$ is a rational function;

(2) $\phi(u)$ belongs to $\mathbb{C}(\exp (\alpha z))$ for some $\alpha$ in $\mathbb{C}$, i.e., $\phi(u)$ is a rational function of an exponential;

(3) $\phi(u)$ belongs to $\mathbb{C}\left(\wp(z), \wp^{\prime}(z)\right)$ for some Weierstrass $\wp$-function, i.e., $\phi(u)$ is an elliptic function.

The assumption that $\phi(u)$ satisfies an algebraic addition theorem in a domain $D$ implies that $\phi(u)$ satisfies a first order algebraic differential equation there. Under the hypotheses of Theorem 1, we can 
apply the Big Picard Theorem to conclude that either $\phi(u)$ is a rational function, or there are infinitely many pairs of points at which $\phi(u)$ and $\phi^{\prime}(u)$ have the same values. Since $\phi(u)$ satisfies a first order algebraic differential equation, it follows that the higher derivatives of $\phi(u)$ are rational functions of $\phi(u)$ and $\phi^{\prime}(u)$. If $\phi(u)$ is not rational, there are infinitely many pairs of points at which $\phi(u)$ and all its derivatives have the same values, that is, at which the coefficients in the Taylor expansions of $\phi(u)$ are the same. One can now conclude that either $\phi(u)$ is rational or it is periodic. In the latter case a similar argument applies to the restriction of $\phi(u)$ to a period strip to show that $\phi(u)$ is either a rational function of an exponential or it is doubly periodic. This establishes the theorem.

Next we drop the assumption that $\phi(u)$ is meromorphic in the plane. We assume instead only that $\phi(u)$ is holomorphic in a neighborhood of the origin and satisfies an algebraic addition theorem there. Let $\boldsymbol{\Phi}$ be the complete analytic function determined by $\phi(u)$, let $W$ be the Riemann surface of $\boldsymbol{\Phi}$, and let $\tau: W \rightarrow \mathbb{C}$ be the natural projection of $W$ to the complex plane.

One can use the addition formula to show that the Riemann surface $W$ is spread over the entire complex plane, and that the only singularities of $\Phi$ are poles and algebraic branch points. An intricate argument then shows that $\phi(u+v)$ is a root of a polynomial equation $H(\phi(u+v))=0$ whose coefficients are single valued functions of $u+v$. One can further prove that the coefficients have algebraic addition theorems - this turns out to be a matter of eliminating the right things from a system of algebraic equations. Thus, the lemma applies to the coefficients. Another argument by elimination shows that $\boldsymbol{\Phi}$ is an algebraic function of an appropriately chosen coefficient of $H$, and this establishes the version of Weierstrass's theorem stated in $\S 3$.

\section{REFERENCES}

[A] Lars V. Ahlfors, Complex Analysis, McGraw-Hill, New York, 1979.

[C] K. Chandrasekharan, Elliptic Functions, Springer-Verlag, Berlin, 1985.

[CH] D. R. J. Chillingworth and J. Hubbard, A note on nonrigid Nash structures, Bull. Amer. Math. Soc., 77 (1971), 429-431.

[DK] M. Delfs and M. Knebusch, Locally Semialgebraic Spaces, Lecture Notes in Mathematics, 1173, Springer-Verlag, Berlin, 1985.

[H] Harris Hancock, Lectures on the Theory of Elliptic Functions, Analysis, Dover, New York, 1958.

[NP] Ali Nesin and Anand Pillay, Some model theory of compact Lie groups, preprint. 
[NPR] Ali Nesin, Anand Pillay, and Vladimir Razenj, Groups of dimension two and three over o-minimal structures, preprint.

[P] Anand Pillay, On groups and fields definable in o-minimal structures, J. Pure Applied Algebra, 53 (1988), 239-255.

[PS] A. Pillay and C. Steinhorn, Definable sets in ordered structures, I, Trans. Amer. Math. Soc., 295 (1986), 565-592.

[R] Vladimir Razenj, One dimensional groups over an o-minimal structure, preprint.

[Ro] Robert Robson, Embedding semi-algebraic spaces, Mathematische Zeitschrift, 183 (1983), 365-370.

[S] Masahiro Shiota, Nash Manifolds, Lecture Notes in Mathematics, 1269, Springer-Verlag, Berlin, 1987.

[Si] C. L. Siegel, Topics in Complex Function Theory, Vol. 1, Elliptic Functions and Uniformization Theory, Wiley-Interscience, New York, 1969.

Received March 12, 1991.

LOUISIANA STATE UNIVERSITY

BATON Rouge, LA 70803

AND

INDIANA UNIVERSITY

SOUTH BEND , IN 46634 


\section{PACIFIC JOURNAL OF MATHEMATICS EDITORS}

\author{
V. S. VARADARAJAN \\ (Managing Editor) \\ University of California \\ Los Angeles, CA 90024-1555 \\ Herbert Clemens \\ University of Utah \\ Salt Lake City, UT 84112 \\ F. Michael Christ \\ University of California \\ Los Angeles, CA 90024-1555 \\ THOMAS ENRIGHT \\ University of California, San Diego \\ La Jolla, CA 92093
}

\author{
Nicholas ERcolani \\ University of Arizona \\ Tucson, AZ 85721 \\ R. FINN \\ Stanford University \\ Stanford, CA 94305 \\ VAughan F. R. JONES \\ University of California \\ Berkeley, CA 94720 \\ C. C. Moore \\ University of California \\ Berkeley, CA 94720 \\ MARTIN ScharlemanN \\ University of California \\ Santa Barbara, CA 93106 \\ Harold Stark \\ University of California, San Diego \\ La Jolla, CA 92093
}

STEVEN KeRCKHOFF

Stanford University

Stanford, CA 94305

\begin{tabular}{|c|c|c|c|c|}
\hline \multicolumn{5}{|c|}{ ASSOCIATE EDITORS } \\
\hline R. ARENS & $\begin{array}{l}\text { E. F. BECKENBACH } \\
(1906-1982)\end{array}$ & NEUMANN & $\begin{array}{c}\text { F. WoLF } \\
(1904-1989)\end{array}$ & K. Yoshida \\
\hline \multicolumn{5}{|c|}{ SUPPORTING INSTITUTIONS } \\
\hline \multicolumn{2}{|c|}{ UNIVERSITY OF ARIZONA } & \multicolumn{3}{|c|}{ UNIVERSITY OF OREGON } \\
\hline \multicolumn{2}{|c|}{ UNIVERSITY OF BRITISH COLUMBIA } & \multicolumn{3}{|c|}{ UNIVERSITY OF SOUTHERN CALIFORNIA } \\
\hline \multicolumn{2}{|c|}{ CALIFORNIA INSTITUTE OF TECHNOLOGY } & \multicolumn{3}{|c|}{ STANFORD UNIVERSITY } \\
\hline \multicolumn{2}{|c|}{ UNIVERSITY OF CALIFORNIA } & \multicolumn{3}{|c|}{ UNIVERSITY OF HAWAII } \\
\hline \multicolumn{2}{|c|}{ MONTANA STATE UNIVERSITY } & \multicolumn{3}{|c|}{ UNIVERSITY OF TOKYO } \\
\hline \multicolumn{2}{|c|}{ UNIVERSITY OF NEVADA, RENO } & \multicolumn{3}{|c|}{ UNIVERSITY OF UTAH } \\
\hline & \multicolumn{3}{|c|}{ WASHINGTON STATE UNIVERSITY } \\
\hline \multicolumn{2}{|c|}{ OREGON STATE UNIVERSITY } & \multicolumn{3}{|c|}{ UNIVERSITY OF WASHINGTON } \\
\hline
\end{tabular}

The Supporting Institutions listed above contribute to the cost of publication of this Journal, but they are not owners or publishers and have no responsibility for its content or policies.

Mathematical papers intended for publication in the Pacific Journal of Mathematics should be in typed
form or offset-reproduced (not dittoed), double spaced with large margins. Please do not use built up fractions
in the text of the manuscript. However, you may use them in the displayed equations. Underline Greek letters
in red, German in green, and script in blue. The first paragraph must be capable of being used separately as
a synopsis of the entire paper. In particular it should contain no bibliographic references. Please propose a
heading for the odd numbered pages of less than 35 characters. Manuscripts, in triplicate, may be sent to any
one of the editors. Please classify according to the 1991 Mathematics Subject Classification scheme which
can be found in the December index volumes of Mathematical Reviews. Supply name and address of author
to whom proofs should be sent. All other communications should be addressed to the managing editor, or
Elaine Barth, University of California, Los Angeles, California $90024-1555-05$.
There are page-charges associated with articles appearing in the Pacific Journal of Mathematics. These
charges are expected to be paid by the author's University, Government Agency or Company. If the author or
authors do not have access to such Institutional support these charges are waived. Single authors will receive
50 free reprints; joint authors will receive a total of 100 free reprints. Additional copies may be obtained at
cost in multiples of 50 .

The Pacific Journal of Mathematics (ISSN 0030-8730) is published monthly except for July and August. Regular subscription rate: $\$ 190.00$ a year (10 issues). Special rate: $\$ 95.00$ a year to individual members of supporting institutions.

Subscriptions, orders for numbers issued in the last three calendar years, and changes of address should be sent to Pacific Journal of Mathematics, P.O. Box 969, Carmel Valley, CA 93924, U.S.A. Old back numbers obtainable from Kraus Periodicals Co., Route 100, Millwood, NY 10546.

The Pacific Journal of Mathematics at P.O. Box 969, Carmel Valley, CA 93924 (ISSN 0030-8730) is published monthly except for July and August. Second-class postage paid at Carmel Valley, California 93924, and additional mailing offices. Postmaster: send address changes to Pacific Journal of Mathematics, P.O. Box 969, Carmel Valley, CA 93924.

PUBLISHED BY PACIFIC JOURNAL OF MATHEMATICS, A NON-PROFIT CORPORATION Copyright (C) 1992 by Pacific Journal of Mathematics 


\section{Pacific Journal of Mathematics}

\section{Vol. 154, No. $2 \quad$ June, 1992}

Manuel (Rodriguez) de León, J. A. Oubiña, P. R. Rodrigues and

Modesto R. Salgado, Almost $s$-tangent manifolds of higher order . . . . 201

Martin Engman, New spectral characterization theorems for $S^{2} \ldots \ldots \ldots 215$

Yuval Zvi Flicker, The adjoint representation $L$-function for GL $(n) \ldots \ldots 231$

Enrique Alberto Gonzalez-Velasco and Lee Kenneth Jones, On the range

of an unbounded partly atomic vector-valued measure . ............ 245

Takayuki Hibi, Face number inequalities for matroid complexes and

Cohen-Macaulay types of Stanley-Reisner rings of distributive

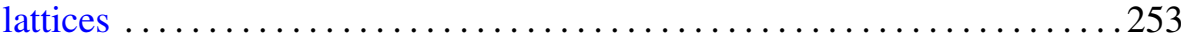

Hervé Jacquet and Stephen James Rallis, Kloosterman integrals for skew

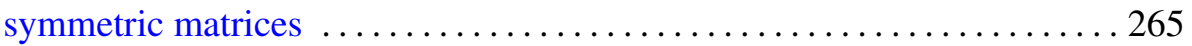

Shulim Kaliman, Two remarks on polynomials in two variables .........285

Kirk Lancaster, Qualitative behavior of solutions of elliptic free boundary problems ..........................................297

Feng Luo, Actions of finite groups on knot complements . ........... 317

James Joseph Madden and Charles Madison Stanton, One-dimensional

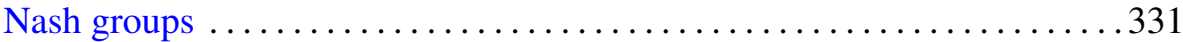

Christopher K. McCord, Estimating Nielsen numbers on

infrasolvmanifolds ......................................... 345

Gordan Savin, On the tensor product of theta representations of $\mathrm{GL}_{3}$ 369

Gerold Wagner, On means of distances on the surface of a sphere. II.

(Upper bounds) 